\title{
Программные и аппаратные преобразования ближнего поля в соответствии с требованиями к измерениям в свободном пространстве систем связи 5G
}

\author{
Б. Дерат ${ }^{1}$, К. Роуэлл ${ }^{2}$, А. Танкелюн ${ }^{3}$, С. Шмитц ${ }^{4}$
}

УДК 621.317 | ВАК 05.11 .01

\begin{abstract}
Повышение пропускной способности систем мобильной связи 5С требует развертывания базовых станций с поддержкой технологии Massive MIMO, базовой сети и мобильных терминалов, поддерживающих работу как в диапазонах ниже 6 ГГц, так и в диапазоне миллиметровых волн. Динамическое формирование диаграммы направленности (ДН) и отсутствие в испытуемых устройствах (ИУ) ВЧ-портов делают проведение измерений в свободном пространстве важнейшей задачей при развертывании систем связи 5С. К счастью, есть решения для выполнения таких измерений, использующие программные и аппаратные преобразования ближнего поля, которые способны справиться с этими задачами.
\end{abstract}

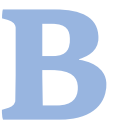
недрение систем связи 5G New Radio (NR) позволит увеличить пропускную способность сетей мобильной радиосвязи, которые работают в диапазоне до б ГГц, называемом также диапазоном частот 1 (FR1) в спецификации 3GPP, или в диапазоне миллиметровых волн (FR2). Новые технологические методы, утвержденные рабочей группой ЗGРP и представителями промышленности, обеспечивают расширение используемой полосы частот с одновременным снижением эксплуатационных расходов.

Основная инновационная деятельность, организуемая для решения задач в диапазоне FR1, направлена на внедрение технологии Massive MIMO на стороне базовых станций (БС) [1]. Системы связи 4G используют однопользовательскую технологию МІMO,

Отдел разработки систем и проектных работ компании

Rohde \& Schwarz в г. Мюнхен, Германия,

benoit.derat@rohde-schwarz.com.

Отдел разработки систем и проектных работ компании

Rohde \& Schwarz в г. Мюнхен, Германия,

corbett.rowell@rohde-schwarz.com.

Отдел разработки систем и проектных работ компании

Rohde \& Schwarz в г. Мюнхен, Германия,

adam.tankielun@rohde-schwarz.com.

Отдел разработки систем и проектных работ компании

Rohde \& Schwarz в г. Мюнхен, Германия,

sebastian.schmitz@rohde-schwarz.com. в рамках которой мобильная станция (MC) выполняет расчет обратной канальной матрицы для выделения отдельных потоков данных. Многопользовательская технология MIMO (MU-MIMO), используемая в системах связи 5G, позволяет перенести эти вычисления из MC в БC с помощью матрицы предварительного кодирования. При этом каждый поток данных независимо принимается отдельным приемником. Формирование диаграммы направленности (ДН) с помощью антенной решетки, которая содержит от 64 до 512 элементов, позволяет снизить помехи соседним абонентским устройствам в схеме MU-MIMO. Помимо повышения пропускной способности, достигаемого при использовании схемы MU-MIMO, технология формирования ДН обладает и другими преимуществами. Пониженное энергопотребление позволяет снизить общие расходы на эксплуатацию сетей путем назначения выделенных каналов и лучей ДН отдельным МС.

Системы связи, работающие в диапазоне FR2, используют широкие полосы пропускания в диапазонах 28 и 39 ГГц. Обратной стороной является увеличение потерь распространения, уровень которых превышает 60 дб на 1 м, и высокий показатель поглощения электромагнитного излучения близлежащими объектами. Как и в случае с системами FR1, решение заключается в использовании антенных решеток и управлении направлением ДН антенны, увеличивающем коэффициент усиления на стороне Мс и на стороне сети. 
Для развертывания как FR1-, так и FR2-систем необходимы производительные высокоинтегрированные решения, в которых объединяются модем, ВЧ-тракт и антенны. В связи с тем, что тестовые ВЧ-порты встречаются в оборудовании все реже, и технологии управления направлением ДН антенны требуют проведения испытаний на уровне системы, основной задачей становится определение новых методов и создание испытательных установок для выполнения анализа рабочих характеристик.

\section{ПОКАЗАТЕЛИ, ИСПОЛЬЗУЕМЫЕ ДЛЯ ИЗМЕРЕНИЙ В СВОБОДНОМ ПРОСТРАНСТВЕ}

Учитывая сказанное, необходимо измерять характеристики антенны и приемо-передатчика - эффективную изотропно излучаемую мощность (EIRP), полную мощность излучения (TRP), эффективную изотропную чувствительность (EIS), полную изотропную чувствительность (TIS), модуль вектора ошибки (EVM), коэффициент утечки мощности в соседний канал (ACLR), спектральную маску излучения (SEM) - в сборе путем измерений в свободном пространстве (OTA, Over-the-Air). Анализ этих характеристик на базе измерений в свободном пространстве играет определяющую роль при выборе требуемого измерительного расстояния.

Характеристики антенны, как правило, измеряются в дальней зоне (FF). Для ИУ Massive MIMO высотой 75 см, излучающего на частоте 2,4 ГГц, с учетом критерия FHD (зона Фраунгофера, $\mathrm{R} \geq 2 \mathrm{D}^{2} / \lambda$, где $\mathrm{R}$ - расстояние от фазового центра антенны; D - размер антенны; $\lambda$ - длина волны), измерения в дальней зоне должны проводиться в камере на расстоянии не менее 9 м. Даже для смартфона высотой 15 см, передающего данные на частоте 43,5 ГГц, требуемое расстояние составляет 6,5 м. Соблюдение этого расстояния позволяет создать рабочую зону (QZ, quiet zone), в которой воздействующее поле имеет, насколько это возможно, равномерное распределение, и где может быть получена плоская волна с отклонением фазы менее $22,5^{\circ}$.

Теоретические исследования [2] показывают, что поведение, характерное для поля в дальней зоне (FF) в направлении максимума главного лепестка, может проявляться намного ближе, чем зона Фраунгофера. Эти результаты доказывают, например, что оценка таких критериев, как EIRP или EIS в дальней зоне для ИУ высотой 15 см, излучающего на частоте 24 ГГц, может быть выполнена на расстоянии всего 1,14 м. Уменьшение расстояния на 70\% по сравнению с расстоянием, необходимым для измерений в дальней зоне, достигается ценой увеличения ошибки по долготе, вызываемой отклонением фазового центра от центра системы координат, которая используется при измерении. Кроме того, на небольших расстояниях невозможно выполнение точных измерений уровней боковых лепестков [3].
Несмотря на то, что такие прямые измерения в дальней зоне на небольших расстояниях подходят не для всех задач, есть причины для измерений данным способом в случаях, когда условия применения проверены и подтверждены. Это связано с высокими расходами на постройку и эксплуатацию громоздких безэховых камер, используемых для выполнения измерений в свободном пространстве, и ограничениями динамического диапазона. Условия проведения таких измерений могут соблюдаться в случае так называемого белого ящика, где известны местоположение антенны в устройстве и размер ее апертуры.

\section{ПРОГРАММНЫЕ МЕТОДЫ ПРЕОБРАЗОВАНИЯ ПОЛЯ БЛИЖНЕЙ ЗОНЫ}

Выполнение прямых измерений в дальней зоне в случае "белого ящика" может оказаться невозможным в случаях, если размер излучающей апертуры выходит за пределы рабочей зоны, если неизвестна точная позиция антенны в ИУ или если несколько антенн, расположенных на противоположных краях ИУ, выполняют одновременную передачу. В этом случае мы должны предположить протекание поверхностных излучающих токов в любой части ИУ и воспользоваться подходом, известным как «черный ящик».

Эффективным подходом к выполнению таких измерений в небольших помещениях является использование программных методов преобразования поля ближней зоны (NF) в дальнюю зону (NFFF, near-field to far-field transformations), при которых размер рабочей зоны перестает иметь значение. Реализации математических моделей NFFF могут различаться, однако общая концепция остается неизменной: как минимум для двух компонентов поляризации электромагнитного поля (Е, Н или их комбинации) выполняется измерение амплитуды и фазы на поверхности, охватывающей ИУ.

Результаты измерений обрабатываются для пересчета поля на большие расстояния и получения компонентов излучения в дальней зоне согласно принципу Гюйгенса. Альтернативные методы преобразования базируются на методах аппроксимации поля дальней зоны сферическими волновыми функциями, плоскими волновыми функциями или решении интегральных уравнений наряду с методами, применяемыми для улучшения скорости и точности вычислений с учетом таких параметров, как частота пространственной дискретизации и область сканирования или усечения

На рис. 1 представлен пример коммерческой системы, позволяющей проводить прямые NF- и FF-измерения с выполнением сферического сканирования в окрестности ИУ и использующей сферический позиционер (conical cut positioner). В этой системе ИУ расположено на поворотной платформе, способной вращаться в азимутальной плоскости, тогда как двухполяризационная антенна 


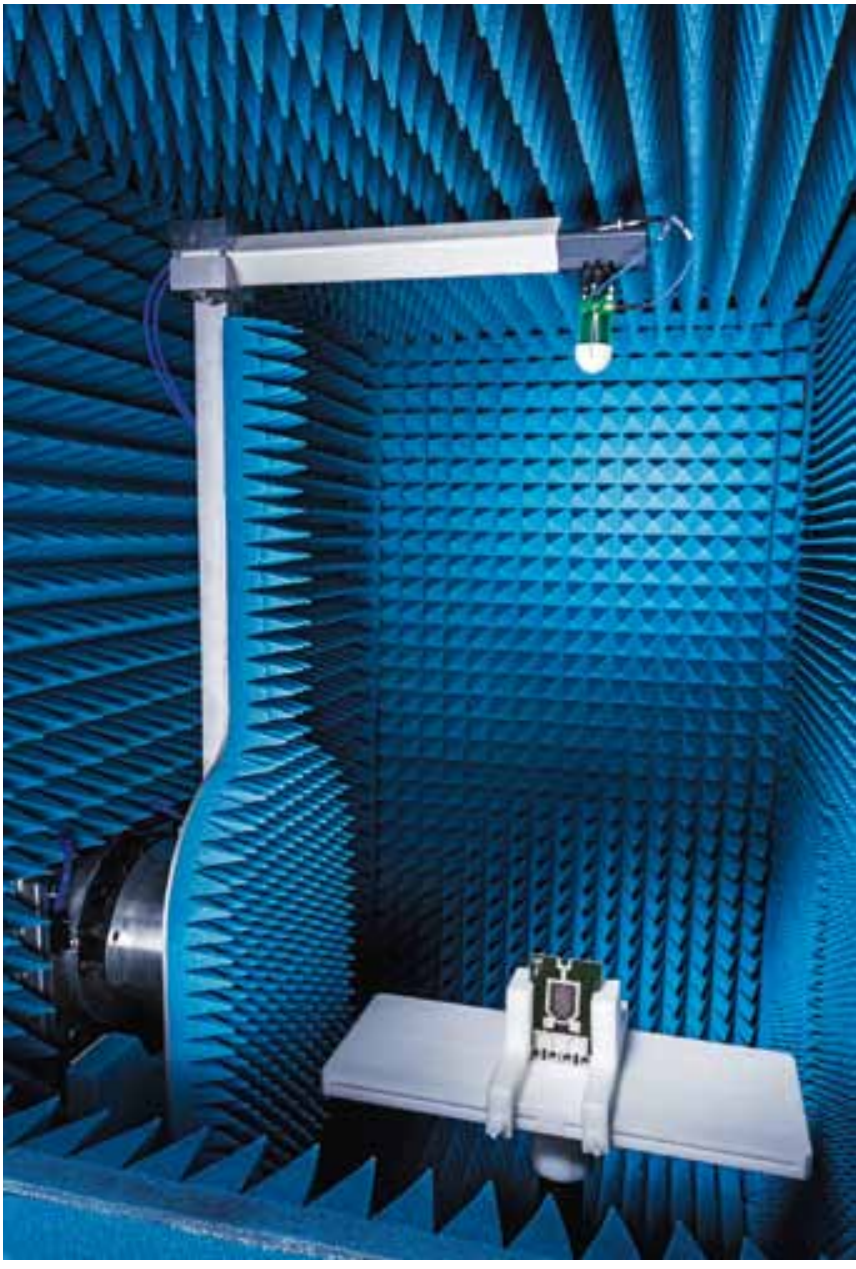

Рис. 1. Пример системы измерения, позволяющей выполнять сферическое сканирование и поддерживающей функцию программного преобразования ближнего поля: измерение параметров антенной решетки, работающей на частоте 28 ГГц, с помощью испытательной системы R\&S ATS1000

Вивальди установлена на головке штатива, вращающегося в угломестной плоскости. В рассматриваемом случае ИУ оборудовано тестовыми ВЧ-портами, что позволяет подключить его к одному из портов векторного анализатора цепей (ВАЦ). Порты измерительной антенны соединены с двумя другими разъемами ВАЦ, что позволяет выполнять анализ поля в ближней зоне (NF) в рамках измерения комплексных S-параметров

Методы измерения поля в ближней зоне зачастую опираются на следующие основополагающие допущения, применимые к вышеописанному сценарию испытания пассивной антенны:

- доступен входной разъем (фланец, порт) испытуемой антенны для подачи возбуждающего Вч-сигнала, одновременно используемого в качестве опорного для фазовых измерений;
- ВЧ-сигнал представляет собой немодулированный сигнал (CW);

- применим принцип взаимности, согласно которому ДН в передающем режиме (Тx) и приемном режиме $(\mathrm{Rx})$ на одной и той же частоте одинаковы.

B передающем режиме измерений (Тх) доступны процедуры, для которых эти допущения не обязательны. Например, были предложены методы для ИУ, излучающих модулированный сигнал при отсутствии доступа к ВЧ-входу антенны. Реализации аппаратных средств и средств обработки, применяемых для получения информации о фазе распространяемых сигналов, могут различаться. В них, например, могут быть задействованы интерферометрические методы или многопортовые фазово-когерентные приемники [4], при этом в качестве источника опорной фазы используется отдельная антенна. В случае систем, подобных представленной на рис. 1, такая антенна, как правило, крепится на поворотной платформе, которая вращается на азимутальном столе. Альтернативными подходами являются бесфазные методы, при использовании которых информация о фазе может быть получена из результатов измерения амплитуды.

Приемный режим (Rx), однако, более сложен. Во-первых, принцип взаимности не может быть применен к мобильным и базовым станциям, поскольку ВЧ-компоненты приемного тракта в общем случае отличаются от ВЧ-компонентов передающего тракта. Кроме того, в случае с ИУ, не оборудованными тестовыми портами, мощность, доступная на приемном входе Вч-тракта и создаваемая падающей волной от вспомогательной антенны (в данном примере используемой в качестве передатчика), не может быть непосредственно рассчитана в ближней зоне. То есть невозможно отделить приемные характеристики Иу в дальней зоне от влияния испытательной установки на поле в ближней зоне. Кроме того, в этом случае отсутствует доступ к информации об опорной фазе, что делает невозможным применение программного преобразования NFFF. Таким образом, с помощью программного преобразования NFFF может быть точно рассчитан критерий EIRP, однако вычисление показателя EIS невозможно.

\section{ЗАДАЧИ ИЗМЕРЕНИЯ ХАРАКТЕРИСТИК ПРИЕМОПЕРЕДАТЧИКОВ В БЛИЖНЕЙ ЗОНЕ}

Еще одной важной задачей измерений в свободном пространстве является анализ таких показателей приемопередающей радиостанции, как EVM, ACLR или SEM. Методы программных преобразований NFFF предназначены для обработки повторяющихся сигналов, то есть ВЧ-несущих, которые определяют параметры распространения. Однако эти части сигнала не представляют интереса с точки зрения анализа вышеперечисленных критериев, для оценки которых необходимо извлечение информации из параметров модуляции несущей. 
Первая трудность заключается в том, что эти показатели сильно зависят от значения отношения сигнал-шум (SNR) на стороне приемника (анализатор спектра в режиме Tх или ИУ в режиме Rx). Эта проблема может быть решена путем выполнения предварительной оценки полной трехмерной ДН в режиме передачи (Tx) или приема (Rx) для определения направления максимума главного лепестка. После этого может быть выполнена демодуляция и, например, измерение EVM в указанной области. Однако вопрос достоверности полученных данных и их соответствия результатам для поля в дальней зоне остается открытым. В случае одиночного приемопередатчика

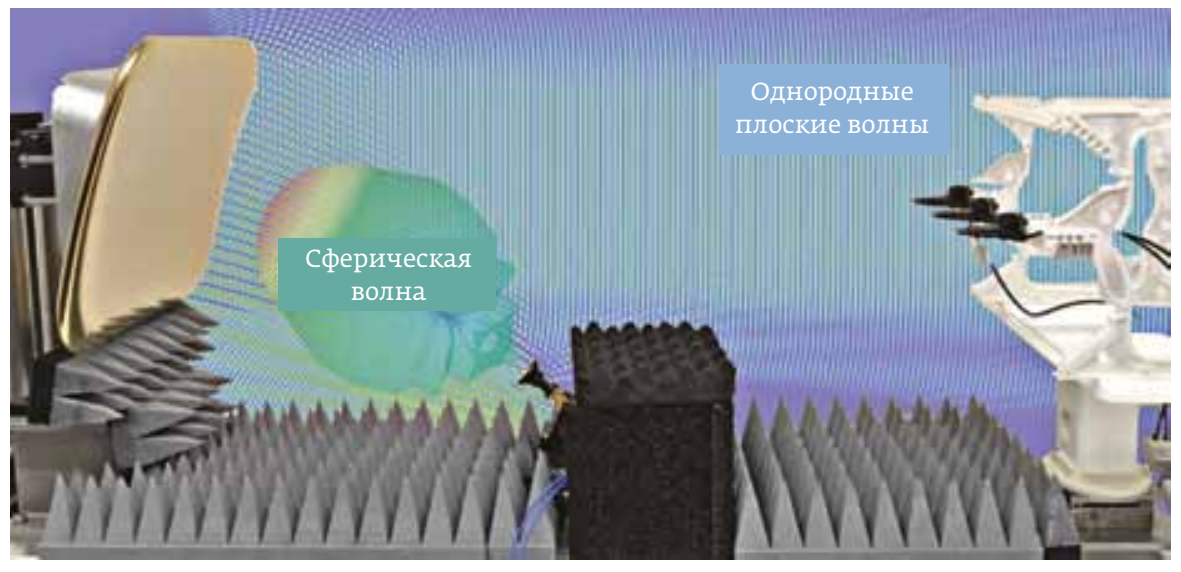

Рис. 2. Пример установки CATR с рефлектором с закругленными краями, позволяющей выполнить преобразование сферического волнового фронта в плоский волновой фронт (поля рассчитаны с использованием модели фактической испытательной установки, реализованной в ПО CST MWS для частоты 28 ГГц) значение EVM в ближней зонедолжно быть равно значению EVM в дальней зоне при условии, что значение SNR превышает определенный порог, определяемый схемой модуляции (например, выше 20 дБ). В случае нескольких независимых одновременно работающих приемопередатчиков значение EVM в ближней зоне может не иметь непосредственной связи со значением EVM в дальней зоне, что объясняется позиционной зависимостью коэффициента шума для поля в ближней зоне.

\section{АППАРАТНЫЕ ПРЕОБРАЗОВАНИЯ ПОЛЯ В БЛИЖНЕЙ ЗОНЕ - ОТВЕТ НА ВСЕ ВОПРОСЫ}

Альтернативные методы испытания подразумевают выполнение измерений в свободном пространстве в ближней зоне с использованием аппаратного преобразования вместо программного. Идея заключается в физическом создании условий дальней зоны в указанной рабочей зоне на небольшом расстоянии. Эти методы также известны как методы квазидальней зоны (indirect FF).

\section{УМЕНЬШЕНИЕ РАЗМЕРОВ СИСТЕМЫ ДЛЯ СОКРАЩЕНИЯ ЭКСПЛУАТАЦИОННЫХ РАСХОДОВ}

В системе CATR (испытание антенн на небольших расстояниях) используется рефлектор для преобразования сферической волны в плоскую, и наоборот. Использование принципа наименьшего времени Ферма позволяет сфокусировать плоскую волну в одной точке с помощью параболического рефлектора. Если измерительная антенна расположена в этой фокальной точке, то с помощью принципа взаимности можно сгенерировать плоскую волну. Это стало возможным благодаря тому, что параболический рефлектор формирует плоский волновой фронт из падающего на него от облучателя

сферического фронта (рис. 2) в области, где расположено ИУ (рабочая зона).

В основе появления ошибок в системе CATR лежат две основные причины: геометрическая конфигурация рефлектора с краевыми эффектами и качеством обработки поверхности, ограничивающими частотный диапазон, и характеристики облучателя.

Если рефлектор построен с использованием простой параболической секции, наличие острых краев приводит к появлению дифракции, которая значительно ухудшает рабочую зону в связи с появлением выбросов, уровень которых может достигать 2 дБ. Для устранения этого явления используют такие методы, как нанесение зазубрин и закругление краев, что обеспечивает рассеяние энергии в сторону от рабочей зоны. Размер и форма зазубренных/закругленных краев определяют нижнюю рабочую частоту, тогда как шероховатость поверхности - верхнюю рабочую частоту.

Характеристики ДН облучателя оказывают непосредственное влияние на размер рабочей зоны, поскольку рефлектор, по сути, "проецирует» ДН облучателя в рабочую зону. Размеры рефлектора с зазубренными / закругленными краями, как правило, не менее чем в два раза превышают размеры ИУ/рабочей зоны, тогда как рефлектор с острыми краями - в 3-4 раза больше рабочей зоны. Оптимальное расстояние от ИУ до рефлектора составляет 5/3 фокусного расстояния рефлектора. Оптимальное фокусное расстояние может быть получено из производственных допусков на форму в диапазоне приблизительно 0,3-1 отношения фокусного расстояния к диаметру параболической секции.

Так как размеры рабочей зоны зависят от характеристик рефлектора, а не от расстояния от него, это значительно 


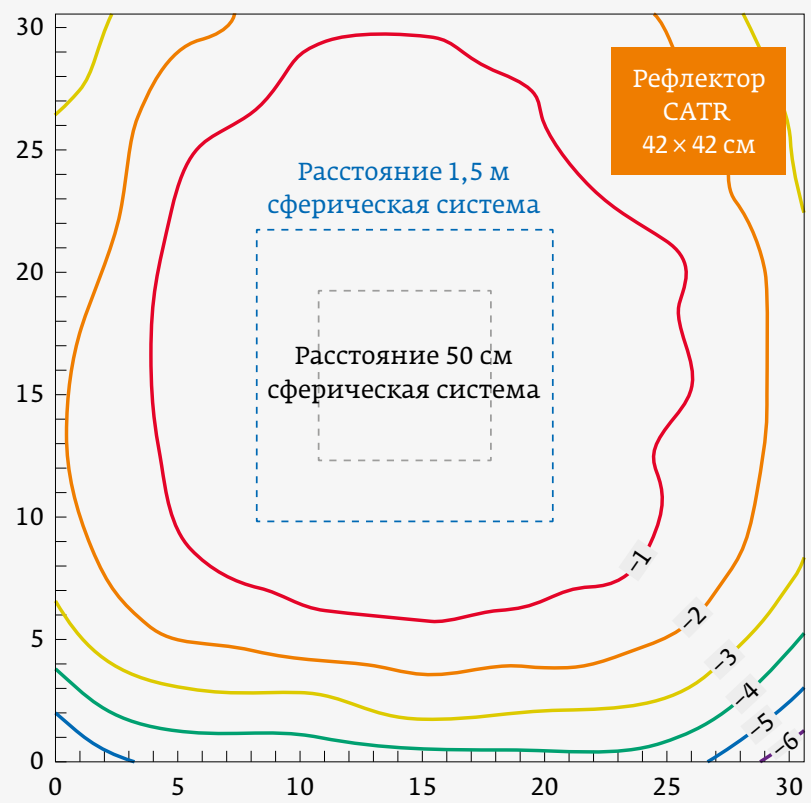

Рис. 3. Сравнение размеров рабочей зоны, измеренных на частоте 28 ГГц при неравномерности амплитуды 2 дБ, для системы CATR с рефлектором $42 \times 42$ см с закругленными краями и систем с прямым измерением FF на расстояниях 50 см и 1,5 м. Изолинии указывают размеры рабочей зоны для одной и той же системы CATR при различных требованиях к неравномерности амплитуды. Вертикальная и горизонтальная шкалы - в см

упрощает решение задачи создания большой зоны молчания в небольших помещениях. На рис. 3 показана измеренная неравномерность амплитуды в рабочей зоне 27 см при частоте 28 ГГц для системы CATR, похожей на представленную на рис. 2, с рефлектором 42×42 см. Эта установка CATR вписывается в небольшую испытательную камеру, размеры которой составляют $2 \times 1,5 \times 0,85$ м. Система для выполнения прямых FF-измерений, которая могла бы обеспечить рабочую зону тех же размеров, потребовала бы работы на расстоянии 14,5 м.

Такие технологии представляют большой интерес в контексте проведения испытаний мобильных или базовых станций, работающих в поддиапазоне FR2 стандарта 5G NR, поскольку позволяют значительно уменьшить размеры тестовой установки. Кроме того, система CATR обладает теми же возможностями, что и система FF, в скорости проведения измерений и позволяет выполнять прямые измерения параметров Вч-приемопередатчиков как в режиме передачи (Tx), так и в режиме приема (Rx). Haконец, поскольку потери в тракте такой системы возникают только в той ограниченной области, где волны распространяются между облучателем и рефлектором, динамический диапазон системы CATR также улучшается по сравнению с подходом, ориентированным на выполнение прямых FF-измерений.

Система CATR, представленная на рис. 3, обладает фокусным расстоянием 0,7 м, тогда как эквивалентное расстояние для FF-систем равно 14 м, в результате чего разница уровней потерь в тракте составляет 26 дБ.

\section{СИНТЕЗ ПЛОСКОЙ ВОЛНЫ: ЭКОНОМИЧЕСКИ ЭФФЕКТИВНАЯ АЛЬТЕРНАТИВА ДЛЯ ВЫПОЛНЕНИЯ ЭФИРНЫХ ИСПЫТАНИЙ БАЗОВЫХ СТАНЦИЙ 5G FR1}

Рефлектор системы CATR, как правило, изготавливается из цельной алюминиевой заготовки, что обеспечивает соответствие строгим требованиям к геометрической конфигурации поверхности. Требования к размерам ИУ для стандарта 5G NR FR2 в миллиметровом диапазоне позволяют применять компактные и легкие рефлекторы (от 20 до 40 кг). При работе в поддиапазоне 6 ГГц FR1 стандарта 5G масса рефлекторов значительно возрастает и может достигать сотен килограмм для тестируемых базовых станций. Стоимость, время изготовления и сложность эксплуатации тяжелых рефлекторов становятся чрезмерными.

Легкой и экономически эффективной альтернативой является использование "электронной версии" рефлектора системы CATR [3]. Объединяя ДН нескольких антенн, формирующих фазированную антенную решетку, и обеспечивая излучение сигналов с управляемыми значениями амплитуды и фазы, можно обеспечить режим плоской волны в заданной рабочей зоне. Версия этого метода фокусировки в ближнем поле использовалась на протяжении нескольких лет для выполнения испытаний больших фазированных антенных решеток RADAR в MIT

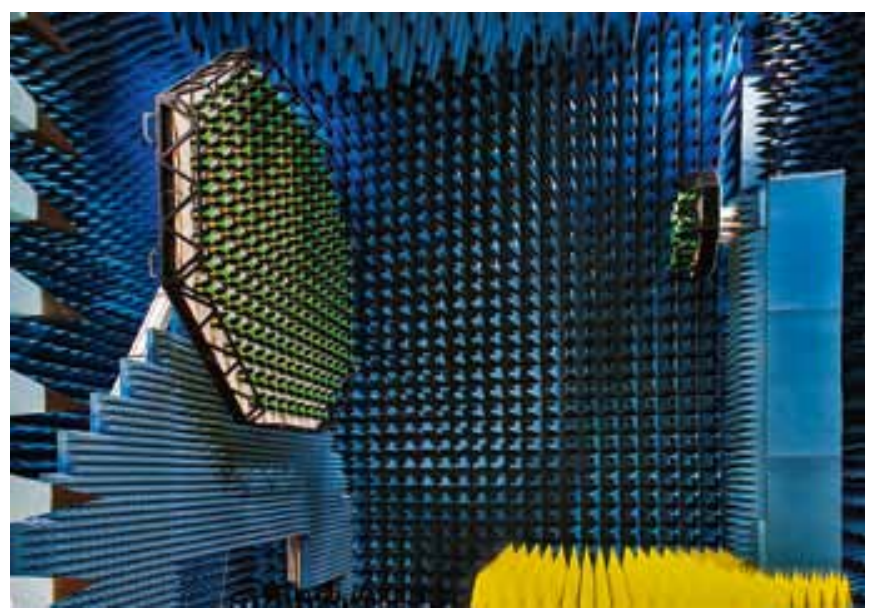

Рис. 4. Система R\&S PWC200 с фазированной антенной решеткой $\mathrm{PWC}$ и калибровочной антенной решеткой, установленной на сферическом позиционере (greatcircle cut positioner) 
Lincoln Labs и была предложена в качестве основной для выполнения измерений в свободном пространстве для БС в стандарте 3GРP [5].

На рис. 4 показана система преобразования плоской волны (plane wave converter, PWC), представленная на конференции European Conference on Antennas and Propagation в 2018 году [3]. Она состоит из 156 широкополосных антенн Вивальди с системой формирования ДН, включающей фазовращатели и аттенюаторы, которые расположены в задней части системы. Эта PWC-система имеет ширину 1,8 м и позволяет создавать сферическую рабочую зону диаметром 1 м на расстоянии всего 1,5 м в частотном диапазоне от 2,3 до 3,8 ГГц. В установке, пока-

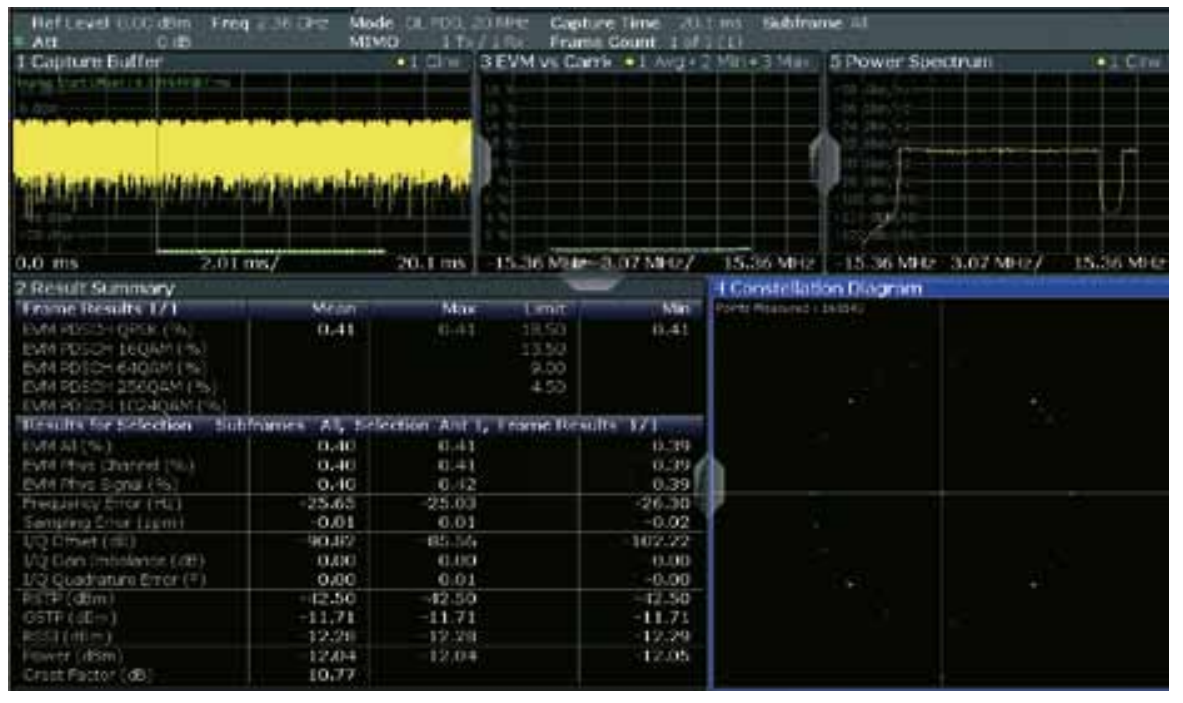

Рис. 5. Результаты измерений EVM для одного сигнала с полосой 20 МГц, полученные с помощью системы R\&S PWC200 при облучении испытуемой антенны пятью сигналами на разных несущих

занной на рис. 4, ИУ (в данном слу-

чае калибровочная антенна) установлено на позиционере скомбинированными поворотными осями, что позволяет выполнять полное сферическое сканирование. Калибровочная антенна используется для расчета соответствующих поправочных коэффициентов для отдельных ВЧ-каналов PWC-системы, а также позволяет определять потери в тракте всей испытательной системы. РWC-система является взаимной и имеет только один ВЧ-вход / выход, который может быть подключен к генератору сигналов, анализатору спектра или ВАЦ, что позволяет проводить испытания устройств, как оборудованных тестовыми ВЧ-портами, так и не оснащенных ими.

На рис. 5 показаны результаты измерения EVM для отдельной несущей, выполненного с помощью PWC-системы для OFDM-сигнала с пятью 20-МГц несущими, который сформирован векторным генератором сигналов R\&S SMW200A в диапазоне от 2,35 до 2,45 ГГц. Для выходной мощности установлено значение 5 дБм, она подается на испытуемую коммутируемую антенную решетку $60 \times 60$ см. Демодуляция выполняется с помощью векторного анализатора сигналов R\&S FSW, подключенного к PWC-системе, измеряющего в полосе 30,72 МГц. Рассчитанное значение EVM составляет всего 0,41\%, что приблизительно соответствует внутреннему значению EVM измерительных приборов. Значения EVM для остальных четырех несущих не превышают 0,5\%; это говорит о том, что значение EVM, добавляемое PWC-системой в испытательную установку, пренебрежимо мало.

$$
\therefore *
$$

Таким образом, методы измерения в ближней зоне, включающие программные преобразования, подходят для расчета показателей EIRP и TRP. Если измерения связаны с приемом или демодуляцией и подразумевают использование нескольких неидентичных ВЧ-приемопередатчиков, применение методов аппаратных преобразований поля, таких как CATR и PWC, позволяет преодолеть ограничения программных преобразований NFFF. Кроме того, эти методы являются компактной и надежной альтернативой прямым FF-измерениям, что делает их выигрышным решением в контексте проведения будущих сертификационных ВЧ-испытаний абонентского оборудования или базовых станций стандарта 3GPP.

\section{ЛИТЕРАТУРА}

1. Chih-Lin I., Rowell C., Han S., Xu Z., Li G., Pan Z. Toward green and soft: a 5G perspective // IEEE Communications Magazine. 2014. V. 52. № 2. P. 66-73.

2. Derat B. $5 G$ antenna characterization in the FF// Proc. Joint IEEE International Symposium on Electromagnetic Compatibility \& Asia-Pacific Symposium on Electromagnetic Compatibility (2018 Joint IEEE EMC \& APEMC). Singapore, 14 to 17 May 2018.

3. Rowell C., Tankielun A. Plane Wave Converter for $5 \mathrm{C}$ massive MIMO basestation measurements // Proc. 12th European Conference on Antennas and Propagation (EuCAP 2018). 9-13 April 2018, London, UK.

4. Derat B., Aberbour L., Quéléver K. et al. A novel technology for fast and accurate specific absorption rate measurement // Proc. Int. Workshop Anten. Tech. (iWAT). March 4-6, 2013, Karlsruhe, Germany.

5. Rohde \& Schwarz, 2D compact range for testing of AAS basestations. - 3GPP TSG RAN WG4 Meeting \#87, R4-1806605. May 2018. 